\title{
A Fine-Grained Visible Light Communication Position Detection System Embedded in One-Colored Light Using DMD Projector
}

\author{
Motoi Kodama and Shinichiro Haruyama \\ Graduate School of System Design and Management, Keio University, 4-1-1 Hiyoshi, Kohoku-ku, Yokohama, \\ Kanagawa 223-8526, Japan \\ Correspondence should be addressed to Motoi Kodama; motoi.kodama@keio.jp
}

Received 7 October 2016; Revised 2 February 2017; Accepted 15 February 2017; Published 21 March 2017

Academic Editor: Maria Bermudez-Edo

Copyright (C) 2017 Motoi Kodama and Shinichiro Haruyama. This is an open access article distributed under the Creative Commons Attribution License, which permits unrestricted use, distribution, and reproduction in any medium, provided the original work is properly cited.

\begin{abstract}
When we consider the Internet of Things (IoT) society that all of the people and things are connected by the Internet and with each other and they can use the variety of services and applications, the development of sensing and communication technology is very important. As one of its key technologies, the visible light communication (VLC) has attracted attention in the point of ubiquity. In this paper, we propose a fine-grained VLC position detection system embedded in one-colored light using Digital Micromirror Device (DMD) projector for new location services in millimeters and report its concept and fundamental experiment using our prototype of the receiver module with Linux single-board computer, Raspberry Pi. In addition, we mention the future application using our system in a clothes shop. Our experimental results show that our system has high accuracy in millimeters and the potential to be more convenient in the future's location services using the VLC with the DMD projector. There was no trial to use a DMD projector as the illumination until now, but our study shows that the DMD projector has an availability of special modulated VLC illumination type beacon in the IoT age.
\end{abstract}

\section{Introduction}

Today, the visible light communication (VLC) such as Light Fidelity (Li-Fi) becomes an active research area to realize the Internet of Things (IoT) society that all of the people and things are connected by the Internet and with each other and they can use the variety of services and applications $[1,2]$. The VLC is an optical wireless communication technology that uses the light that is visible to human. And it is becoming more popular with the spread of LED lights. In this study, we aim to develop new VLC beacon technology idea for next generation's location services.

In the IoT age, a location service technology with high accuracy becomes extremely important. Various location service technologies have been studied until now [3, 4]; Indoor MEssaging System (IMES) [5, 6], Radio Frequency Identifier (RFID), Wireless Fidelity (Wi-Fi), Ultra Wide Band (UWB), Bluetooth, Infrared Ray (IR), the ultrasound, and the VLC using the image sensor. Particularly, the VLC using image sensor is superior to the other technologies in the precision.

Usually, in the VLC, a modulated LED light as the transmitter transmits the information signal to the receiver such as the photodiode detector $[7,8]$ or the image sensor [9-14]. As its applications using the image sensors, many studies were conducted; the survey measurement $[9,10]$, the robot control [11-13], and Intelligent Transport Systems (ITS) [14]. These studies realized the measurement accuracy in centimeters or millimeters.

In general, because the user has always a camera in today's smart phones and tablet devices and their image sensors can be used as the receivers to receive the high special modulated VLC signals, we consider that the VLC has an advantage such as easy communication using ubiquitous lights and image sensors. However, because it takes time ascribable to heavy image processing and low frame rate of their camera, the received VLC signals' speed is limited. And when the image 


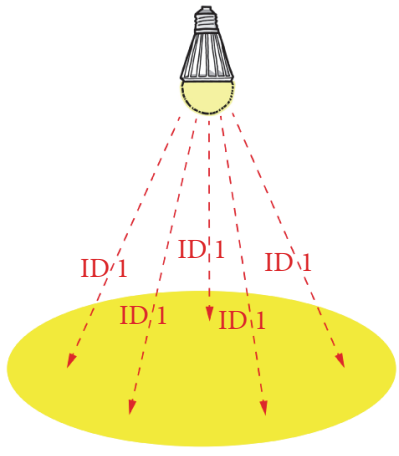

(a)

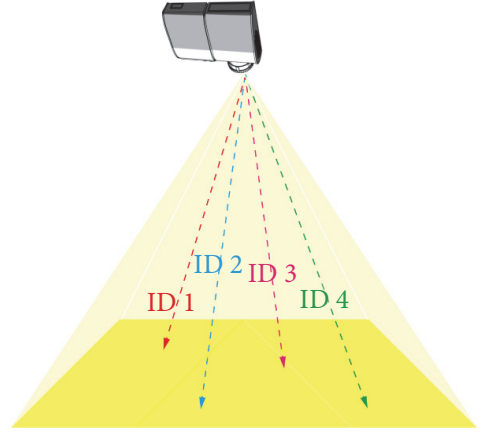

(b)

FIGURE 1: The comparison with VLC system using (a) a LED light and (b) a DMD projector light as the transmitter.

sensor as the receiver is very fast moving, the situation is quite inconvenient to receive the VLC signal precisely.

On the other hand, though the VLC using the photodiode detector is much faster than that using the image sensor due to simple electrical signal processing, single photodiode detector cannot receive multiple VLC signals selectively without the optical signal path's separation. Then, we determine that the purpose of this study is to find the other ways to achieve the high speed and special modulated VLC in such a situation.

As its technological solution, we have focused on the DMD projector [15]. It has been studied to realize the novel displays emitting imperceptible metadata along with the image [16-21]. Our original idea is the application of the DMD projector as the VLC transmitter to achieve the high speed and special modulated VLC beacon illumination for location services in everyday life. Figure 1 shows the comparison with the VLC system using (a) a LED light and (b) a DMD projector light as the transmitter. When a LED light is the transmitter, we can use only one signal in close area. In contrast, when a DMD projector light is the transmitter, it can embed the different information for each pixel and modulate the VLC signals very fast.

In this paper, we propose a fine-grained VLC position detection system embedded in one-colored light using the DMD projector for new location services in millimeters and report its concept and fundamental experiment using our prototype module of the receiver with Linux single-board computer, Raspberry Pi.

In the future, we aim to apply our system to many location services. Figure 2 shows the use case example of our system sending multiplex information in multiple directions using only one DMD projector. As shown in Figure 2, it is possible to obtain different information depending on the collar, the sleeves, and buttons on the same shirt. If you receive the signal around shirt's collar, the receiver shows "This collar is a crew neck." If you receive the signal around shirt's buttons, the receiver shows "Buttons are made of wood." We are developing the VLC technologies to be able to provide a finegrained location service in millimeter order.

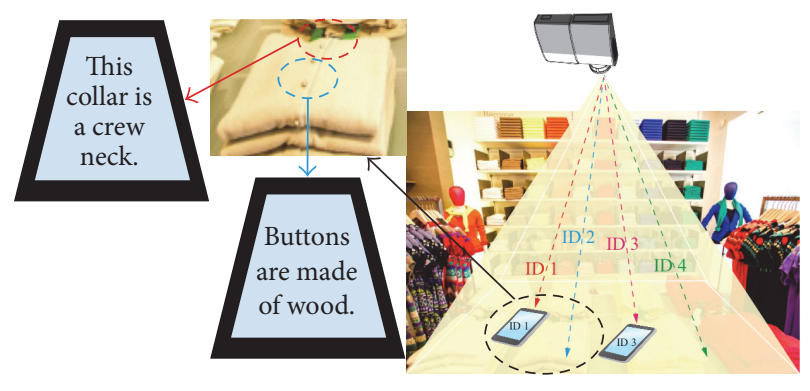

FIGURE 2: The use case example of our system sending multiplex information in multiple directions using only one DMD projector.

The rest of this paper is organized as follows. Section 2 introduces related works of many measurement technologies, in particular, the VLC using the photodiode, the image sensor, and the DMD projector, and shows the comparison with existing location services technology. Section 3 describes materials and methods in our experiment. Section 4 mentions the result and discussion of our experiment. Finally, Section 5 concludes this paper.

\section{Related Works}

This section introduces related works of many measurement technologies.

First, we mention the comparison with existing various indoor location service technologies in the accuracy. Table 1 shows the comparison with existing various location service technologies in the accuracy; IMES, RFID, Wi-Fi, UWB, the ultrasound, IR, and the VLC using the image sensor.

As shown in Table 1, we recognize that the VLC using image sensor is superior to the other technologies in the precision. Next, we explain and discuss individual technical characteristics as the location service aspect.

IMES is the electric wireless technology to get the position signal transmitted from nearest transmitter, which is compatible with Global Positioning System (GPS) signal. 
TABLE 1: The comparison with existing various indoor location service technologies in the accuracy.

\begin{tabular}{lc}
\hline Technology type & Accuracy \\
\hline IMES & Several hundred meters [3] \\
RFID & $1 \mathrm{~m} \sim 5 \mathrm{~m} \mathrm{[4]}$ \\
Wi-Fi & $1 \mathrm{~m} \sim 100 \mathrm{~m} \mathrm{[4]}$ \\
UWB & $1 \mathrm{~cm} \sim 1 \mathrm{~m} \mathrm{[4]}$ \\
Bluetooth & $1 \mathrm{~m} \sim 5 \mathrm{~m} \mathrm{[4]}$ \\
IR & $1 \mathrm{~cm} \sim 5 \mathrm{~m} \mathrm{[4]}$ \\
Ultrasound & $1 \mathrm{~cm} \sim 1 \mathrm{~m} \mathrm{[4]}$ \\
VLC using image sensor & A few millimeters [9-14] \\
\hline
\end{tabular}

TABLE 2: The feature of VLC receivers that are a photodiode detector and an image sensor for the single LED light transmitter.

\begin{tabular}{lcc}
\hline & Photodiode detector & Image sensor \\
\hline $\begin{array}{l}\text { Number of VLC } \\
\text { signals }\end{array}$ & Single & Multiple \\
$\begin{array}{l}\text { Distance } \\
\text { Space resolution }\end{array}$ & A few meters [7] & Very long $(2 \mathrm{~km})[14]$ \\
Signal processing type & $\begin{array}{c}\text { Electrical signal } \\
\text { processing }\end{array}$ & Each pixel \\
\hline
\end{tabular}

However, in fact, IMES gets the position information by using not the calculation of signal sending time difference between the transmitter and the receiver which is GPS method but the identification of transmitter's unique signal. Therefore, IMES is different from the GPS in the signal processing of the receiver side, and it is hard to say that the IMES is the seamless technique that is common in both indoor and outdoor.

RFID is one of the wireless communication technologies in short distance with its dedicated tag for mobile phone, smartphone, and tablet devices which can read the tag using their camera. However, when we use RFID, its tags have to be equipped and the shop staff and so forth will be timeconsuming.

In the way using $\mathrm{Wi}-\mathrm{Fi}$, we can easily use its environment that exists in the wide area. However, an error of several meters at least occurs, and its precision depends on its access point's position and its surroundings delicately.

UWB is the electric wireless communication that its signals spread to broadband in transmitting and receiving, which has several ten centimeters' accuracy. UWB also has to need the setting of the transmitters as with the other electric wireless communication technology.

Bluetooth has several centimeters' accuracy and today's many mobile devices usually equip its receiver module. Though, as its beacon can be getable easily and cheaply, there is some possibility of installing fake beacons, here and there.

The ultrasound and IR transmit the position information similar to the VLC, but they are not visible. Therefore, it is difficult for users to find where the transmitters are fixed, and their systems take a high cost.

In the VLC, there are two major receiver types: the photodiode detector and the image sensor. Table 2 shows the feature of VLC receivers that are a photodiode detector and an image sensor for the single LED light transmitter. From the point of view of spatial multiplex signals, the image sensor is better than the photodiode detector as the receiver. However, as expressed up until now, the VLC using the image sensor is not suitable for high speed VLC communication.

Then, we aim to develop new type VLC technology for location service using the photodiode detector as the receiver, and we focus on the VLC using the DMD projector as the transmitter. In this way, the DMD projector as the transmitter transmits special modulated VLC signals, and the photodiode as the receiver can receive the signal at the high speed.

The VLC using the DMD projector has been actively researched as new information presentation method to users until now, and its accuracy is in millimeters. For example, Kimura et al. made EmiTable [18], which is a tabletop surface pervaded with imperceptible metadata, and Kato et al. made iPvlc [19], which is pixel-level VLC. However, the DMD projector has not been applied for location service yet in previous researches.

The VLC using the image sensor has high accuracy in millimeters. As mentioned in the introduction, its technical problems are heavy image processing and low frame rate of the camera. Though, the VLC using the image sensor is suited to measure the object position that is not moving seriously, and its accuracy is in millimeters.

In this paper, we report its concept and demonstrate its fundamental experiment. We use the DMD projector as new type of VLC indoor location service beacon, which seems to be the illumination at a glance though its lights in several million pixels are modulated very high speed as each pixel VLC signal is distinguished by the position. Our study provides everyone the first idea to realize a fine-grained VLC position detection system embedded in one-colored light using DMD projector, which is high accuracy indoor navigation in the IoT age.

\section{Materials and Methods}

Figure 3 shows a schematic diagram of the VLC experimental system in this study. As shown in Figure 3, we use the system that consists of a Digital Light Processing (DLP) projector (DLP ${ }^{\circledR}$ LightCrafter $4500^{\mathrm{TM}}$ Evaluation Module, Texas Instruments Incorporated) including the DMD as the transmitter and a photodiode detector as the receiver with an analog circuit for frequency filter to eliminate the noise caused by the room light and amplification of the signal and a Raspberry Pi 3 to demodulate the received signals.

The DLP projector can store the continuous binary images of 48 frames and transmit the information of $1140 \times$ 912 pixels arranged in a diamond array configuration for each frame. Figure 4 shows our original coding idea of 48 images including 22 bits' position information created by 2 pulse position modulation (2PPM) and the Gray code; for example, the $x$-coordinate is from 56 to 967 and the $y$-coordinate is from 454 to 1593 . These images are the first 6 flames' header and the remaining 42 frames with the location information to send different signals for each pixel square. As the projector seems to be a flickerless light and the transmitter, we use the $2 \mathrm{PPM}$ to have the same lighting time in all pixels. And we 


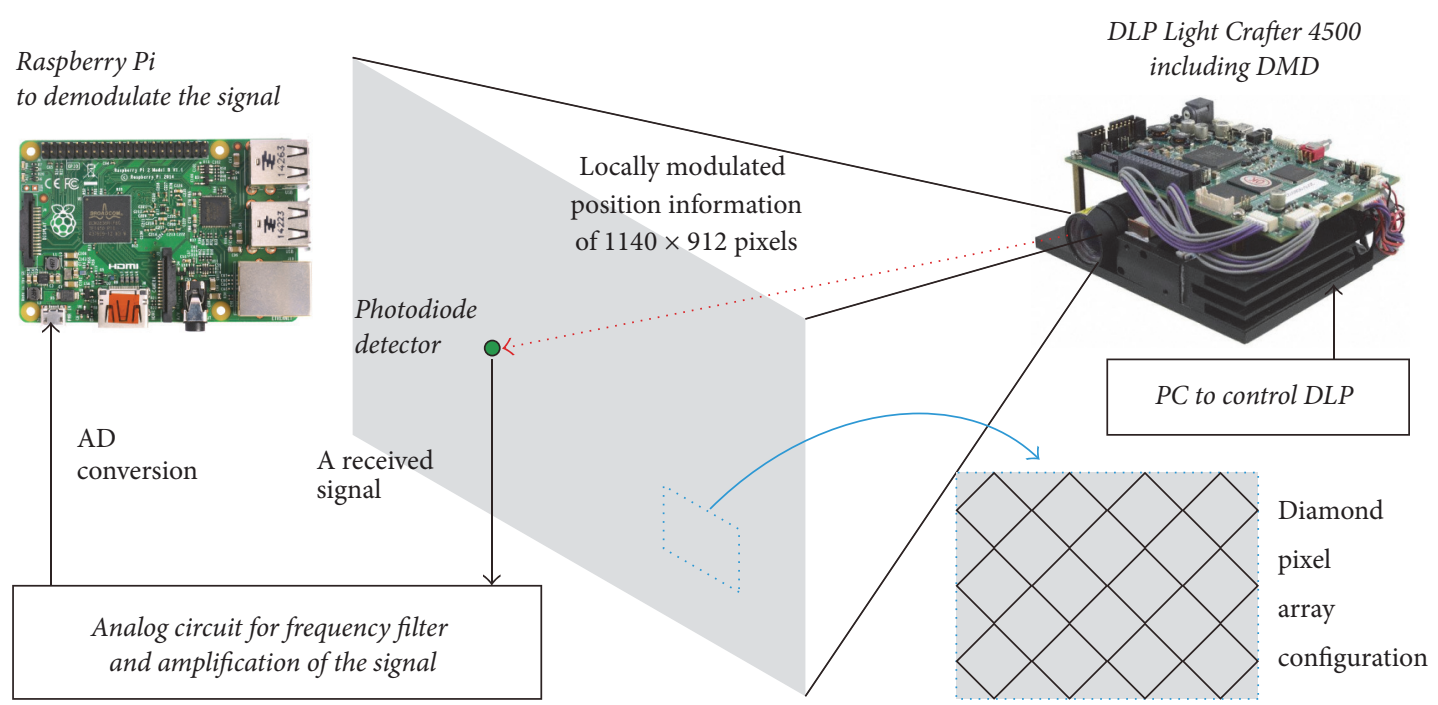

FIGURE 3: A schematic diagram of the experimental system in this study.

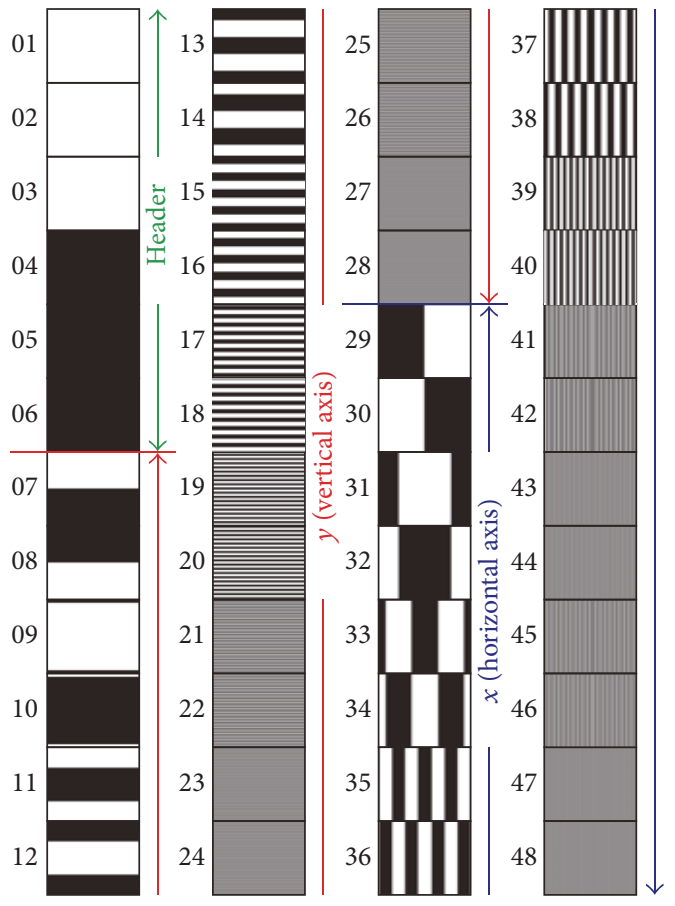

FIgURE 4: Our original coding idea of 48 images including 22 bits' position information created by 2-pulse position modulation (2PPM) and Gray code.

adopted the Gray code whose Hamming distance between adjacent pixels along the vertical axis or the horizontal axis is only one in the boundary of light and darkness to reduce the error in each axis of the images. Therefore, some error was reduced in their error checking process for the signals to confirm that its condition satisfies 2PPM rules.

The signal received by a photodiode detector is input to an analog circuit with a function of frequency filter and the amplification. Before the circuit in the prototype was made, we computed the frequency characteristics in the approximate circuit using LTSpice that is the simulation software for an analog circuit.

Figure 5 shows (a) an approximate circuit diagram and (b) the simulation result of frequency characteristic in the input and the output voltage using LTSpice. In the simulation, to simplify the analysis, we replaced the photodiode detector to an alternating current source of amplitude $1 \mathrm{uA}$. As shown in Figure 5, we obtain a configuration of a frequency filter to eliminate the voltage component in several $\mathrm{Hz}$ or less and several tens $\mathrm{kHz}$ from the received signals.

Then, we created a prototype of the VLC receiver with reference to the calculation results as shown in Figure 5. Figure 6 shows (a) a circuit diagram and (b) a photograph of the VLC receiver with Raspberry Pi 3. The receiver has two channel photodiode detectors and the LCD display to show the position information to the users. Each circuit uses two Operational Amplifiers; one is used for frequency filter and the amplification, and another is used for the adder circuit to process the signals between $0 \mathrm{~V}$ and $5 \mathrm{~V}$ in an analog-todigital converter.

In this study, we have experimentally observed the appearance of the projected image to the human eyes and the position detection accuracy with respect to the horizontal direction and a vertical direction when the 48 signal images are projected from the DLP projector.

Figure 7 shows the photograph of experiment to measure the position detection accuracy when the VLC receiver moves along (a) $x$-axis and (b) $y$-axis of the 48 signal images. The distance between the DLP projector and the receiver was 2.5 meters, because we assumed the distance between the indoor ceiling attached to the light and the floor where the users exist. The receiver was moving every $5 \mathrm{~cm}$ along $x$-axis or $y$-axis from the image edge to measure the signal. 


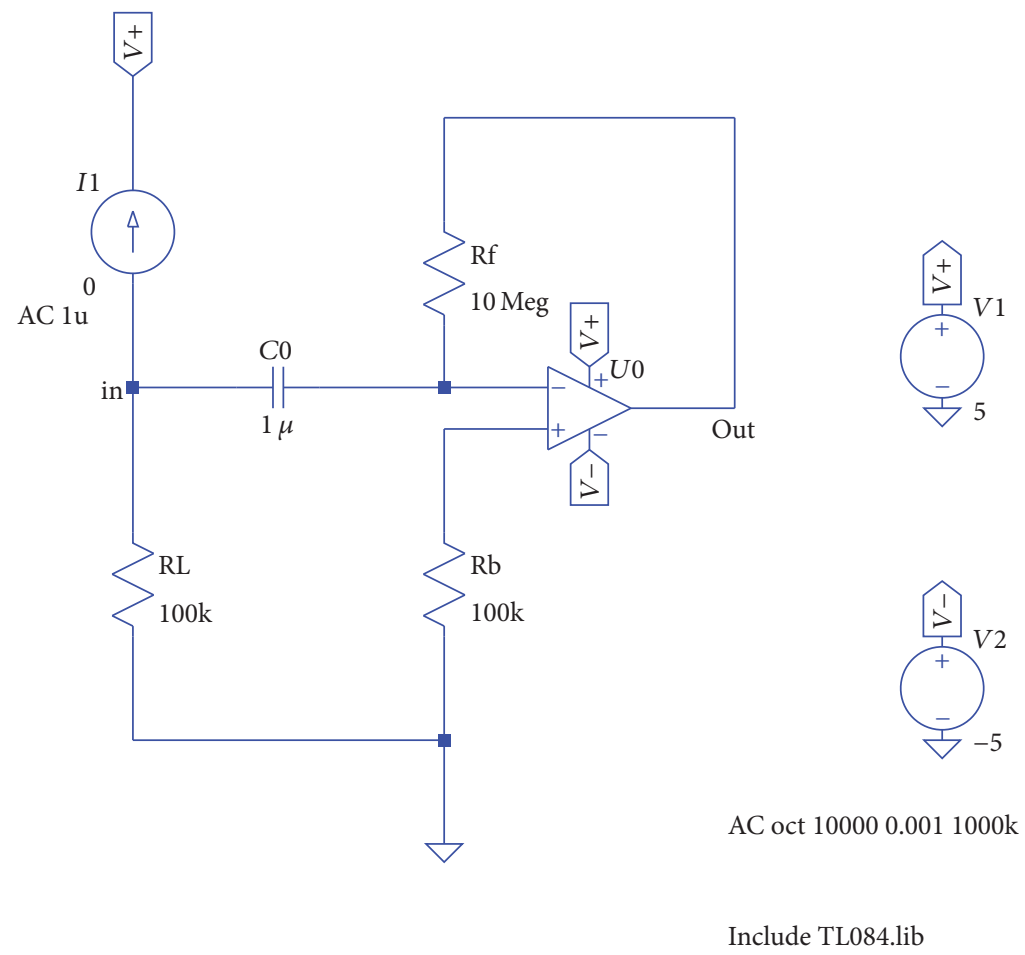

(a)

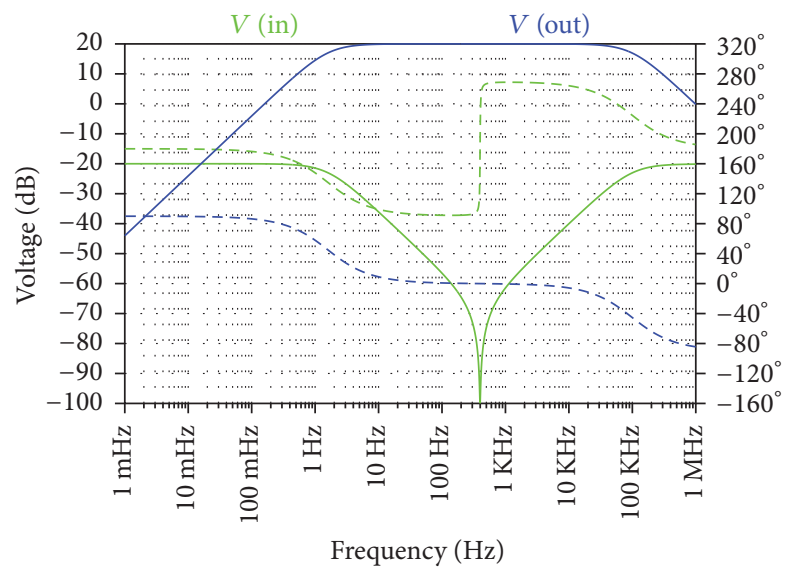

(b)

Figure 5: (a) An approximate circuit diagram and (b) the simulation result of frequency characteristic in the input and the output voltage using LTSpice.

\section{Results and Discussion}

Figure 8 shows the frames of the video projected from the DMD projector on the wall when the frequency of changing frame was (a) $10 \mathrm{~Hz}$ and (b) $4 \mathrm{kHz}$. In Figure 8(a), we watched the projected images one by one. In contrast, because the length of time switching on the light was equal to the length of time switching off the light and the projection frequency was much faster than that human can perceive, the projected images were seen as white illumination as shown in Figure $8(\mathrm{~b})$.

In this study, we collected a questionnaire about its flicker frequency from 5 users. Because the users felt its flicker when the frequency of changing frame was $1.5 \mathrm{kHz}$ with the room lights and at $1.7 \mathrm{kHz}$ without the room lights, we consider that the users can use the DLP projector light whose frequency is higher than $1.7 \mathrm{kHz}$ in our daily life.

Figure 9 shows the received voltage waveform measured in $(x, y)=(76,479)$ and demodulated code by Raspberry Pi 3. As shown in Figure 9, the received voltage waveform has the header signal and the position information with respect to $x$ coordinate and $y$-coodinate in the measured point. Although an error has occurred in a part of the bit, the received signal is demodulated to the position information bits that are more dominant. 


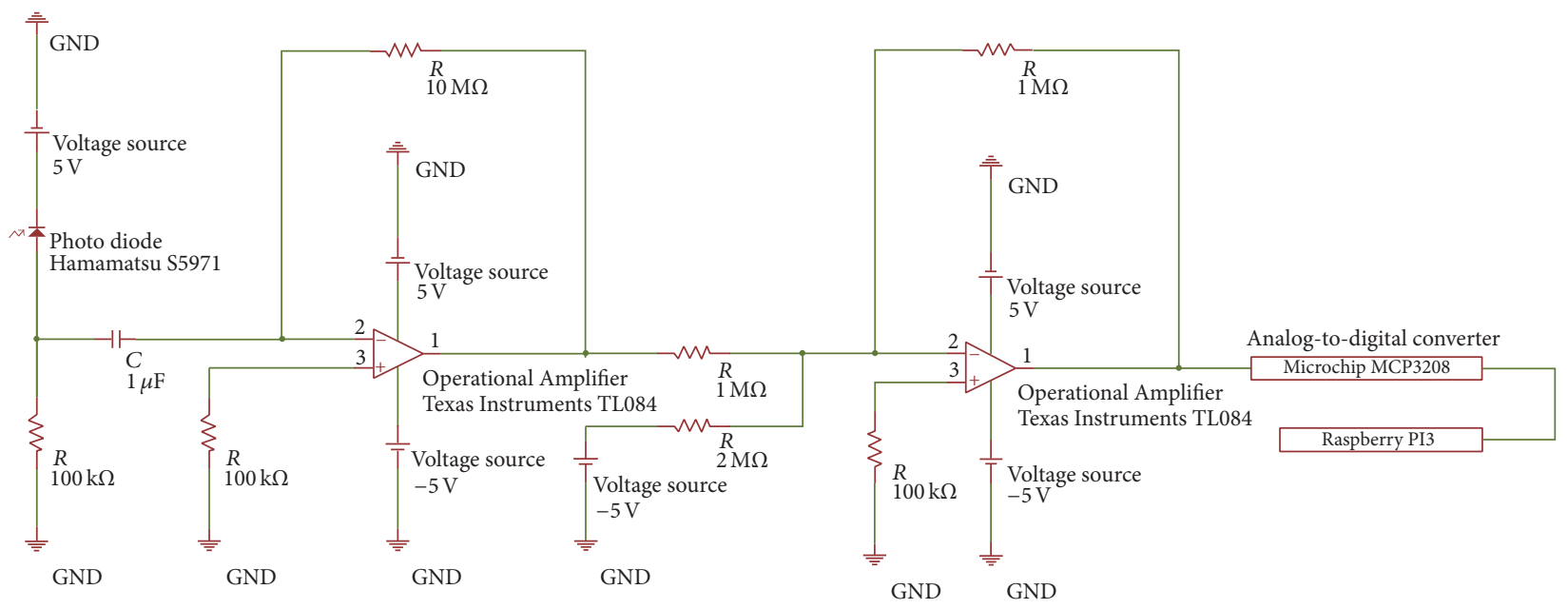

(a)

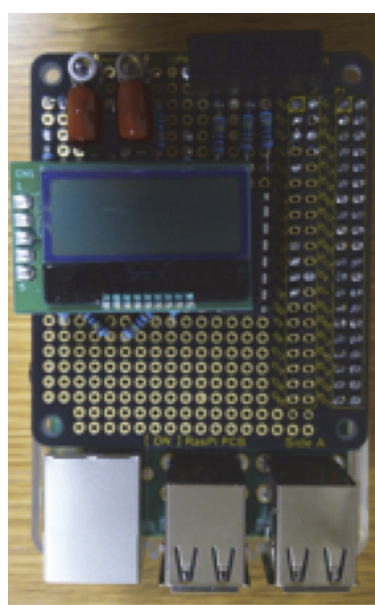

(b)

FIGURE 6: (a) A circuit diagram and (b) a photograph of the VLC receiver with Raspberry Pi 3.

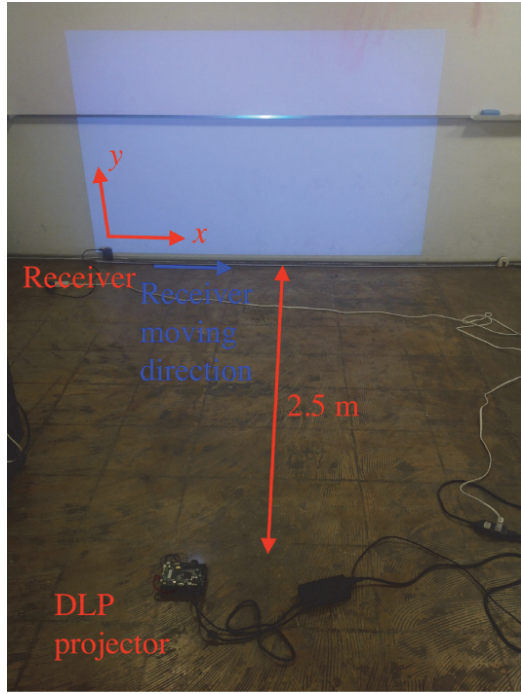

(a)

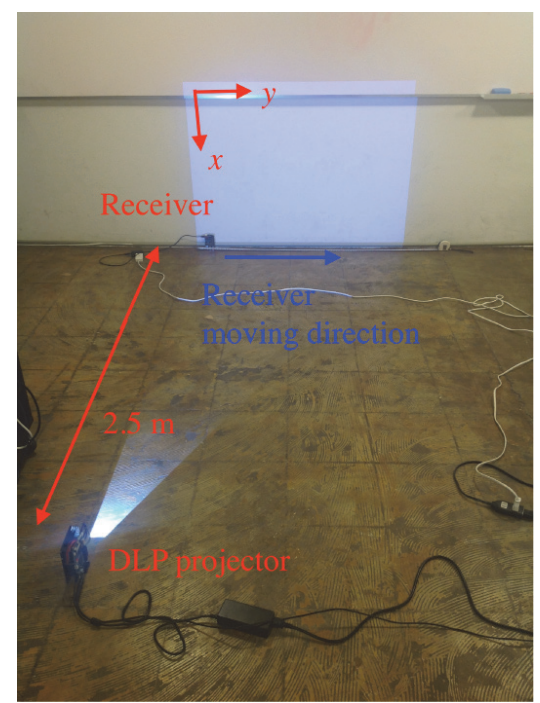

(b)

FIGURE 7: The photograph of experiment to measure the position detection accuracy when the VLC receiver moves along (a) $x$-axis and (b) $y$-axis of the 48 signal images. 


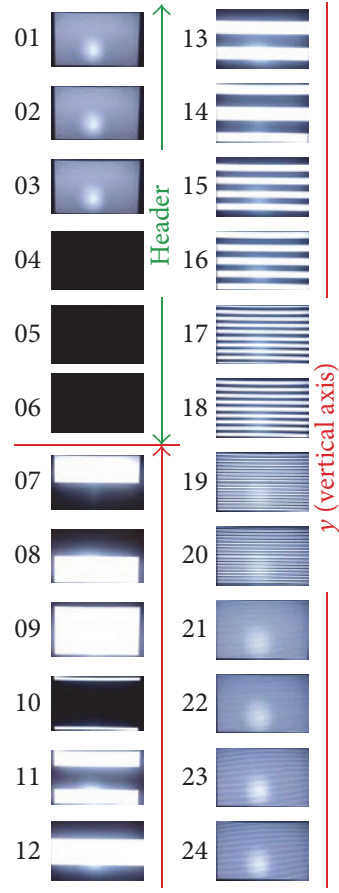

(a)

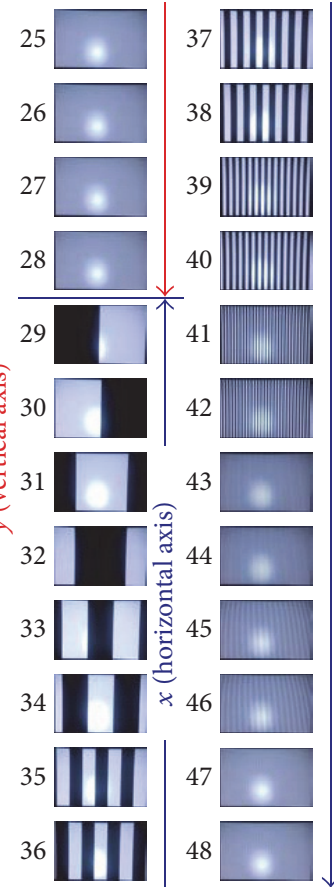

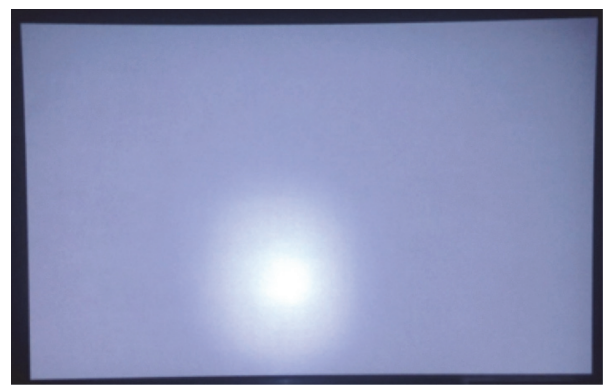

(b)

FiguRE 8: The frames of the video projected from the DMD projector on the wall when the projection frequency of $48 \mathrm{images}$ was (a) $10 \mathrm{~Hz}$ and (b) $4 \mathrm{kHz}$.

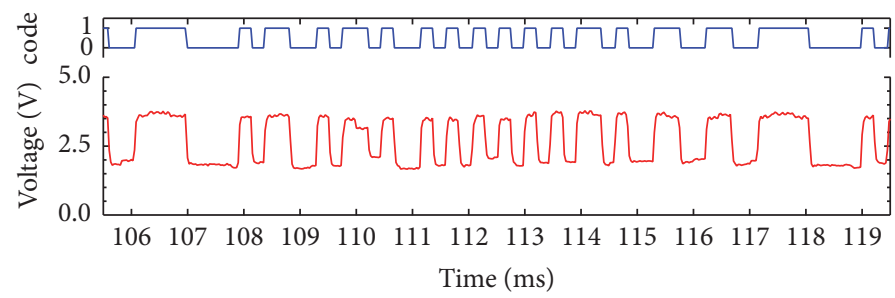

Figure 9: The received voltage waveform measured in $(x, y)=(76,479)$ and demodulated code by Raspberry Pi 3.

Figure 10 shows the photograph of LCD information showed in $(x, y)=(76,479)$ by Raspberry Pi 3 . The LCD display in the receiver indicates the position information obtained from the demodulated signal to the user. In the future, the display will be able to provide useful location services for the user such as the price and features of the product as shown in Figure 2.

Figure 11 shows (a) the received position in the projected images (in pixels), (b) the relationship between $x$ (in pixels range) and the moving distance along $x$-axis, and (c) the relationship between $y$ (in pixels range) and the moving distance along $x$-axis in the measurement when the receiver is moving along the $x$-axis. As shown in Figures 11(a) and 11(c), $y$ in pixel range is in a constant state and is not dependent on the moving distance along $x$-axis that we measured in the range of real space. On the other hand, as shown in Figure 11(b), $x$ in pixel is proportional to moving distance along $x$-axis. Then, we calculated $2.2 \mathrm{~mm} /$ pixel accuracy from the experimental data shown in Figure 11(b).

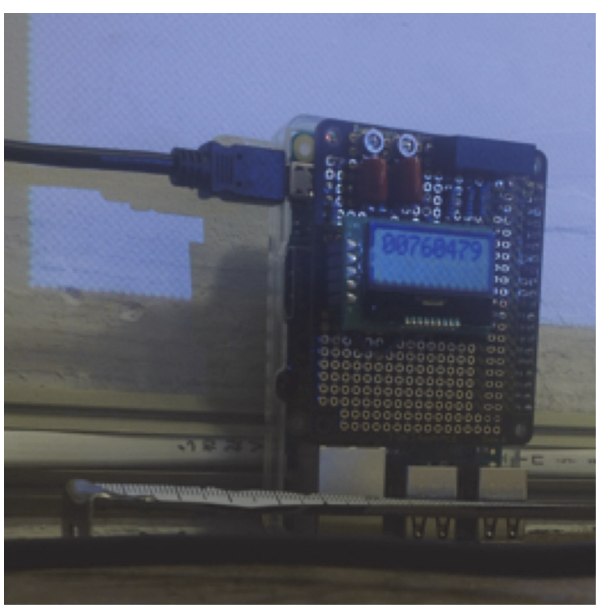

FIGURE 10: The photograph of LCD information showed in $(x, y)=$ $(76,479)$ by Raspberry Pi 3 . 


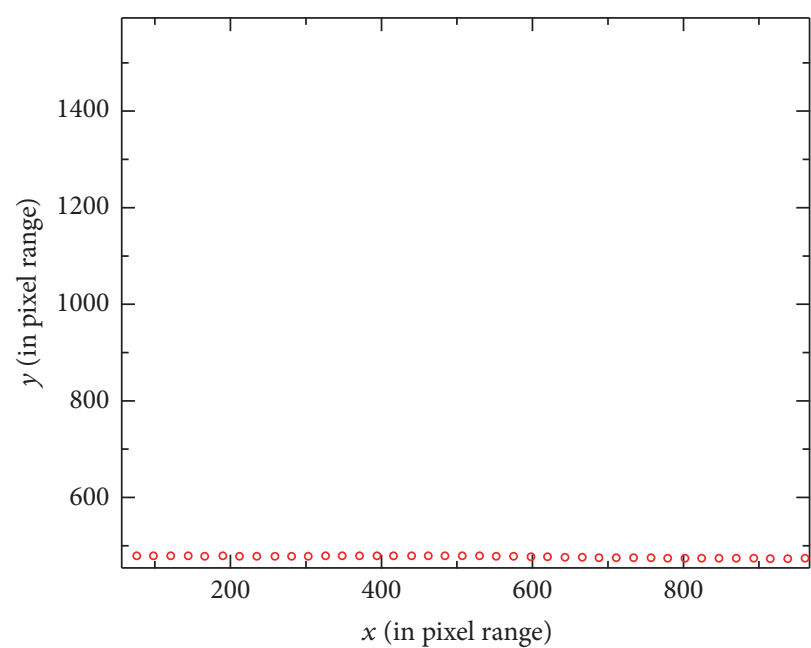

(a)

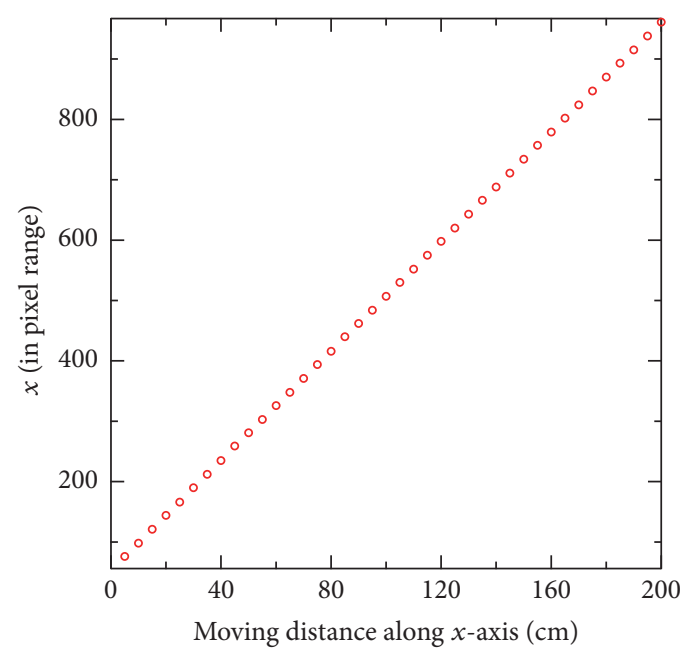

(b)

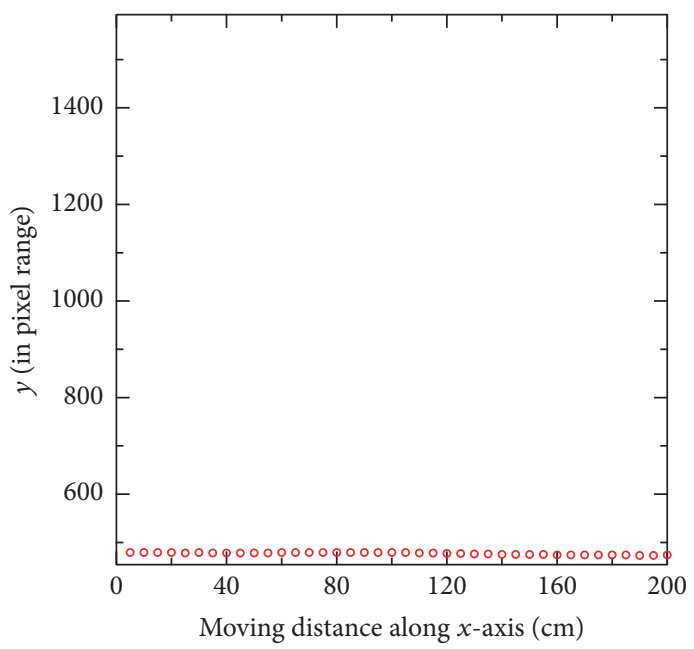

(c)

Figure 11: (a) The received position in the projected images (in pixels), (b) the relationship between $x$ (in pixels range) and the moving distance along $x$-axis, and (c) the relationship between $y$ (in pixels range) and the moving distance along $x$-axis in the measurement when the receiver is moving along the $x$-axis.

Figure 12 shows (a) the received position in the projected images (in pixels), (b) the relationship between $x$ (in pixels range) and the moving distance along $y$-axis, and (c) the relationship between $y$ (in pixels range) and the moving distance along $y$-axis in the measurement when the receiver is moving along the $y$-axis. As shown in Figures 12(a) and 12(b), $x$ in pixel range is in a constant state and is not dependent on the moving distance along $y$-axis that we measured in the range of real space. On the other hands, as shown in Figure 12(c), $y$ in pixel is proportional to moving distance along $y$-axis. Then, we calculated $1.1 \mathrm{~mm} /$ pixel accuracy from the experimental data shown in Figure 12(c).

While these experimental results show that our system has high accuracy in millimeters, the accuracy of the $x$-axis range is a little different from the accuracy of the $y$-axis rage due to the diamond array pixel configuration in the DMD used in this study. Therefore, the accuracy of the vertical and horizontal axes is consistent with the millimeter order; the matter of nonisotropic accuracy can be solved using the checkerboard array of the DMD.

We consider that our system will give the high accuracy VLC projector light beacon in the location services, compared to existing various position detecting technologies.

\section{Conclusions}

In this paper, we propose a fine-grained VLC position detection system embedded in one-colored light using DMD projector for new location services in millimeters and report its concept and fundamental experiment using our original image coding method and our prototype module of the receiver with Raspberry Pi. Our experimental results show that our system has high accuracy in millimeters and the potential to be more convenient in the future's location services using VLC. 


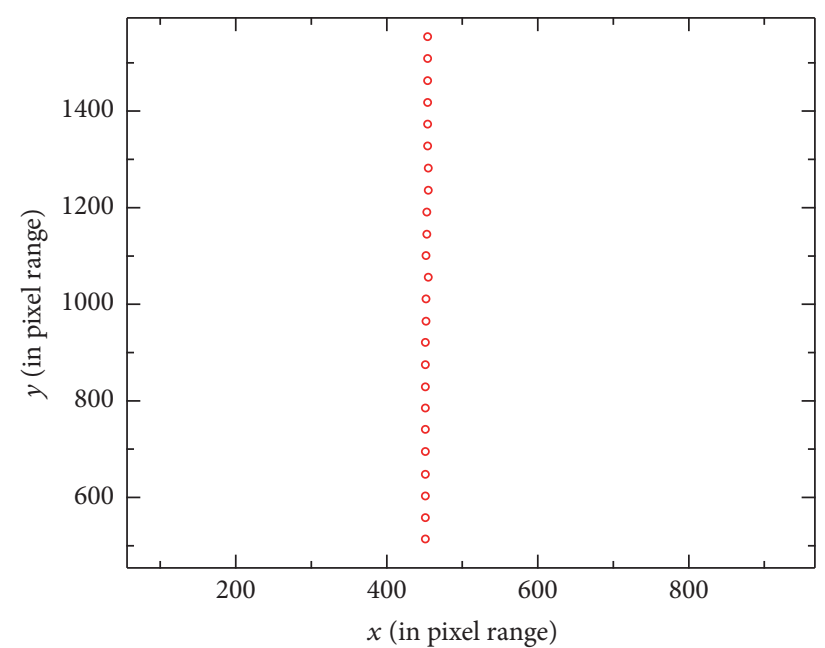

(a)

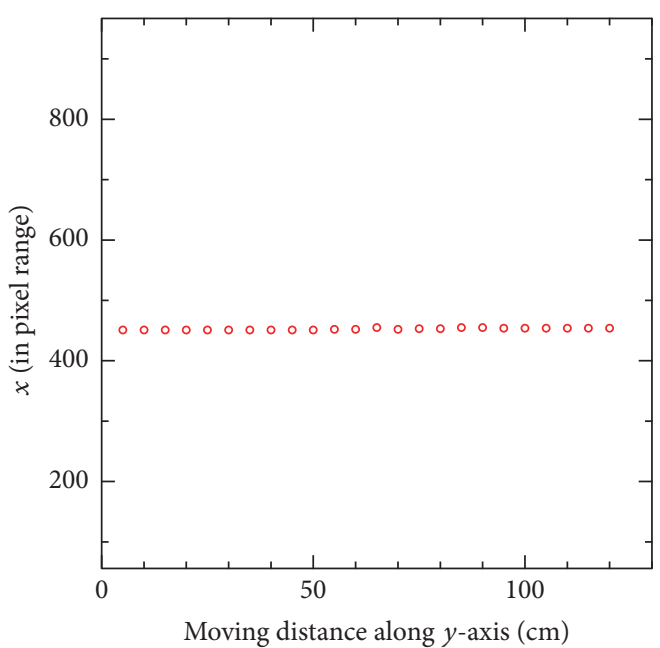

(b)

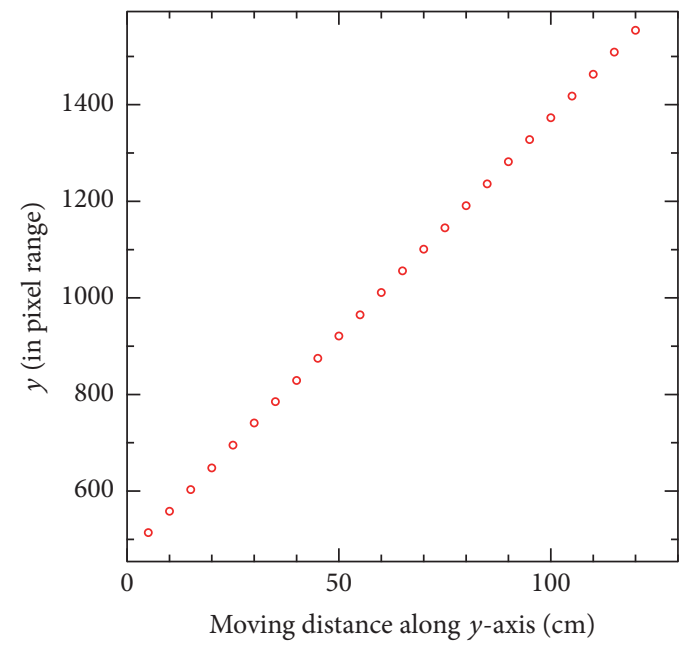

(c)

FIGURE 12: (a) The received position in the projected images (in pixels), (b) the relationship between $x$ (in pixels range) and the moving distance along $y$-axis, and (c) the relationship between $y$ (in pixels range) and the moving distance along $y$-axis in the measurement when the receiver is moving along the $y$-axis.

\section{Conflicts of Interest}

The authors declare that they have no conflicts of interest.

\section{Acknowledgments}

This work was supported by Keio University Doctorate Student Grant-in-Aid Program, Graduate School Recommendation by Graduate School of System Design and Management, "Research and the Application of Transmitter Device for Spatial Modulated Visible Light Communication using Digital Micromirror Device," April, 2014-March, 2017.

\section{References}

[1] D. Tsonev, S. Videv, and H. Haas, "Light fidelity (Li-Fi): towards all-optical networking," in Proceedings of the SPIE 9007 Broadband Access Communication Technologies VIII, 2013.
[2] A. Kumar, A. Mihovska, S. Kyriazakos, and R. Prasad, "Visible light communications (VLC) for ambient assisted living," Wireless Personal Communications, vol. 78, no. 3, pp. 1699-1717, 2014.

[3] G. Deak, K. Curran, and J. Condell, "A survey of active and passive indoor localisation systems," Computer Communications, vol. 35, no. 16, pp. 1939-1954, 2012.

[4] S. Dragan and N. Stojanovic, "Indoor localization and tracking: methods, technologies and research challenges," Facta Universitatis, Series: Automatic Control and Robotics, vol. 13, no. 1, pp. 57-72, 2014.

[5] N. Kohtake, S. Morimoto, S. Kogure, and D. Manandhar, "Indoor and outdoor seamless positioning using indoor messaging system and GPS," in Proceedings of the International Conference on Indoor Positioning and Indoor Navigation (IPIN '11), pp. 21-23, Guimaraes, Portugal, September 2011.

[6] D. Manandhar, S. Kawaguchi, M. Uchida, M. Ishii, and H. Torimoto, "IMES for mobile users social implementation and experiments based on cellular phones for seamless positioning," 
in Proceedings of the International Symposium on GPS/GNSS, pp. 1-8, Tokyo, Japan, 2008.

[7] H. Sugiyama, S. Haruyama, and M. Nakagawa, "Experimental investigation of modulation method for visible-light communications," IEICE Transactions on Communications, vol. 89, no. 12, pp. 3393-3400, 2006.

[8] M. Nakajima and S. Haruyama, "New indoor navigation system for visually impaired people using visible light communication," EURASIP Journal on Wireless Communications and Networking, vol. 2013, no. 1, pp. 1-10, 2013.

[9] H. Uchiyama, M. Yoshino, H. Saito et al., "Photogrammetric system using visible light communication," in Proceedings of the 34th Annual Conference of IEEE Industrial Electronics (IECON '08), pp. 1771-1776, IEEE, Orlando, Fla, USA, November 2008.

[10] H. Mikami, T. Kakehashi, N. Nagamoto, M. Nakagomi, and Y. Takeomi, "Practical applications of 3D positioning system using visible light communication," Reports of Technical Research and Development Center of Sumitomo Mitsui Construction Co. Ltd, vol. 9, pp. 79-84, 2011.

[11] T. Tanaka and S. Haruyama, "New position detection method using image sensor and visible light LEDs," in Proceedings of the 2nd International Conference on Machine Vision (ICMV'09), pp. 150-153, IEEE, Dubai, United Arab Emirates, December 2009.

[12] Y. Nakazawa, H. Makino, K. Nishimori, D. Wakatsuki, and H. Komagata, "Indoor positioning using a high-speed, fish-eye lens-equipped camera in visible light communication," in Proceedings of the International Conference on Indoor Positioning and Indoor Navigation (IPIN '13), October 2013.

[13] Y. Nakazawa, H. Makino, K. Nishimori, D. Wakatsuki, and H. Komagata, "High-speed, fish-eye lens-equipped camera based indoor positioning using visible light communication," in Proceedings of the International Conference on Indoor Positioning and Indoor Navigation (IPIN '15), October 2015.

[14] T. Yamazato, I. Takai, H. Okada et al., "Image-sensor-based visible light communication for automotive applications," IEEE Communications Magazine, vol. 52, no. 7, pp. 88-97, 2014.

[15] M. Kodama and S. Haruyama, "Accurate location service using DMD projector," in Proceedings of the 4th International Conference on Serviceology (ICServ '16), September 2016.

[16] T. Naemura, T. Nitta, A. Mimura, and H. Harashima, "Virtual shadows in mixed reality environment using flashlight-like devices," Transactions of the Virtual Reality Society of Japan, vol. 7, no. 2, pp. 227-237, 2002.

[17] H. Nii, Y. Hashimoto, M. Sugimoto, and M. Inami, "Optical interface using LED array projector," Transactions of the Virtual Reality Society of Japan, vol. 12, no. 2, pp. 109-117, 2007.

[18] S. Kimura, M. Kitamura, and T. Naemura, "EmiTable: a tabletop surface pervaded with imperceptible metadata," in Proceedings of the 2nd Annual IEEE International Workshop on Horizontal Interactive Human-Computer Systems (TABLETOP '07), pp. 189-192, October 2007.

[19] Y. Kato, N. Fukasawa, and T. Naemura, "iPvlc: pixel-level visible light communication for smart mobile devices," in Proceedings of the ACM SIGGRAPH Posters (SIGGRAPH '11), article 45, ACM, Vancouver, Canada, August 2011.

[20] L. Zhou, S. Fukushima, and T. Naemura, "Dynamically reconfigurable framework for pixel-level visible light communication projector," in Emerging Digital Micromirror Device Based Systems and Applications VI, 89790J, vol. 8979 of Proceedings of SPIE, 14 pages, International Society for Optics and Photonics, March 2014.
[21] T. Hiraki, S. Fukushima, and T. Naemura, "Sensible shadow: tactile feedback from your own shadow," in Proceedings of the the 7th Augmented Human International Conference, pp. 1-4, Geneva, Switzerland, Feburary 2016. 

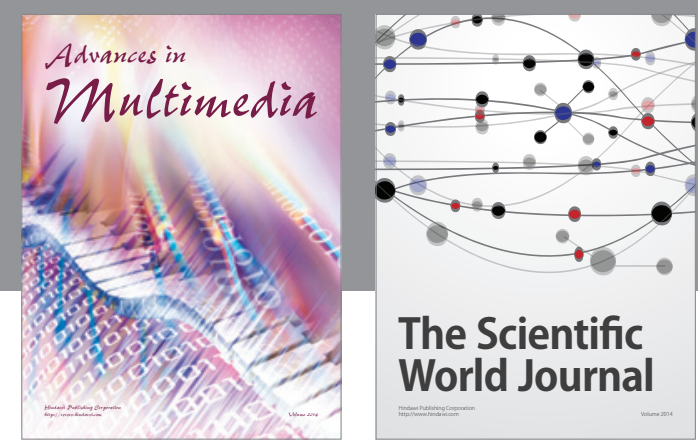

The Scientific World Journal
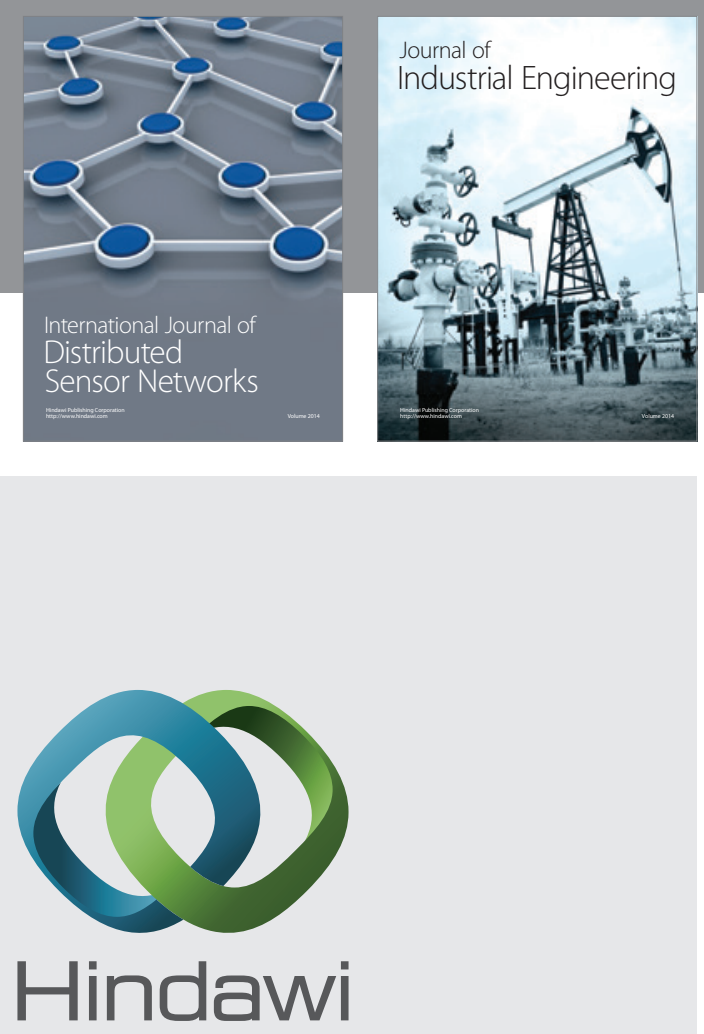

Submit your manuscripts at

https://www.hindawi.com

\section{Computer Networks} and Communications
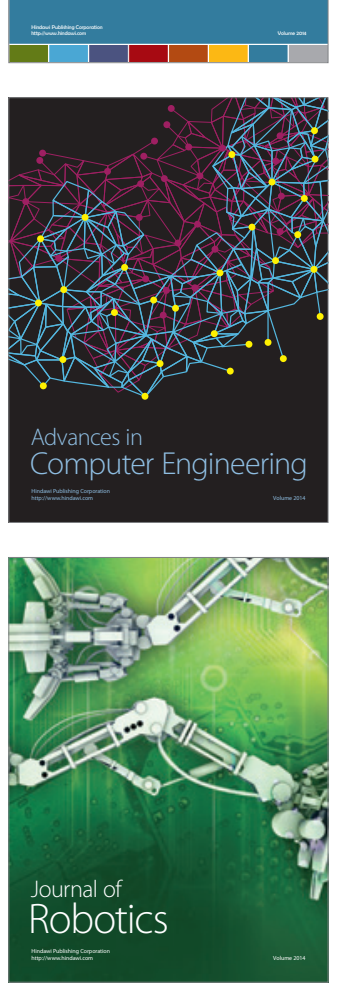
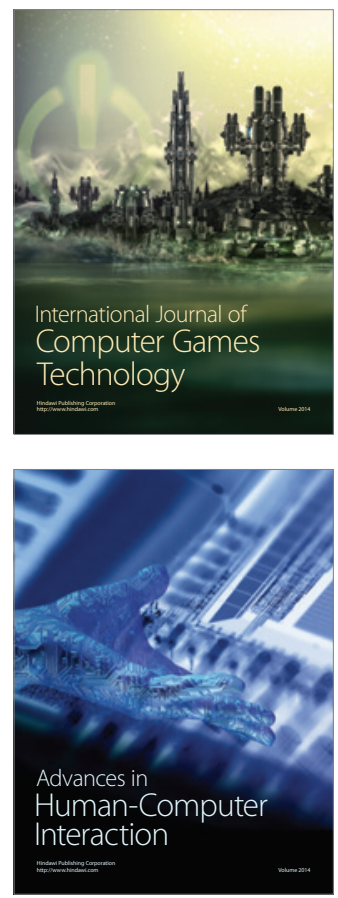
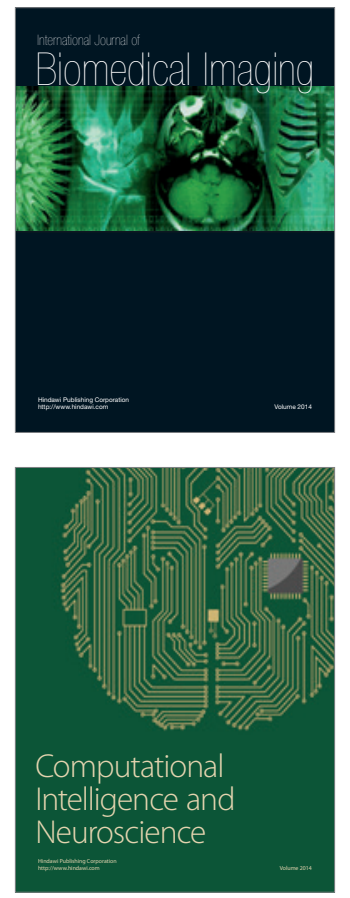
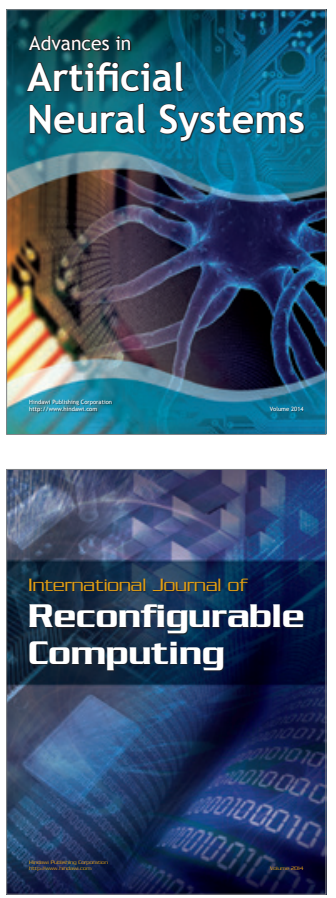
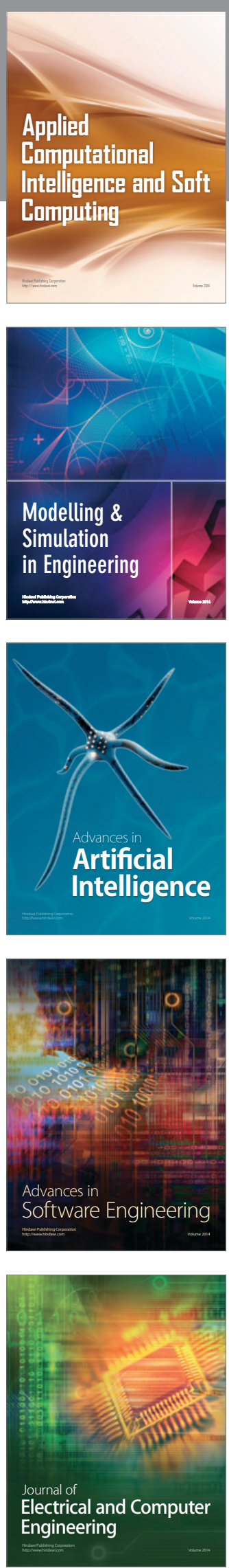